ANNALES

POLONICI MATHEMATICI

$88.3(2006)$

\title{
Canonical tensor fields of type $(p, 0)$ on Weil bundles
}

\author{
by JACEK DęBECKI (Kraków)
}

\begin{abstract}
We give a classification of canonical tensor fields of type $(p, 0)$ on an arbitrary Weil bundle over $n$-dimensional manifolds under the condition that $n \geq p$. Roughly speaking, the result we obtain says that each such canonical tensor field is a sum of tensor products of canonical vector fields on the Weil bundle.
\end{abstract}

Let $A$ be a Weil algebra and $T^{A}$ the Weil functor corresponding to $A$, which is a product preserving bundle functor (see [2]). Fix non-negative integers $n$ and $p$. A canonical tensor field of type $(p, 0)$ on $T^{A}$ is, by definition, a family of tensor fields $V_{M}$ of type $(p, 0)$ on $T^{A} M$ indexed by $n$-dimensional manifolds and satisfying for all such manifolds $M, N$ and every embedding $f: M \rightarrow N$ the condition

$$
\bigotimes^{p} T T^{A} f \circ V_{M}=V_{N} \circ T^{A} f .
$$

Recall that a derivation of the algebra $A$ is a linear map $D: A \rightarrow A$ such that $D(a b)=a D(b)+b D(a)$ for all $a, b \in A$. The vector space of derivations of $A$ will be denoted by Der $A$. It is well known (see [1]) that if $n \geq 1$, then there is a one-to-one correspondence between the canonical tensor fields of type $(1,0)$ (in other words, canonical vector fields) on $T^{A}$ and the derivations of $A$. Namely, every $D \in \operatorname{Der} A$ induces a unique canonical vector field $\widetilde{D}$ on $T^{A}$ such that

$$
\widetilde{D}_{\mathbb{R}^{n}}(X)=\left(D\left(X^{1}\right), \ldots, D\left(X^{n}\right)\right)
$$

for every $X \in A^{n}$, and conversely, for every canonical vector field $V$ on $T^{A}$ there is a unique $D \in \operatorname{Der} A$ such that $V=\widetilde{D}$. Our purpose is to generalize this result to all $p$.

Consider the tensor product $\bigotimes^{p} A$ of the vector spaces $A$. For every $r \in\{1, \ldots, p\}$ and every $a \in A$ we have the linear map $Z_{a}^{r}: \bigotimes^{p} A \rightarrow \bigotimes^{p} A$ such that

2000 Mathematics Subject Classification: Primary 58A32.

Key words and phrases: product preserving bundle functor, Weil algebra. 


$$
Z_{a}^{r}\left(b_{1} \otimes \cdots \otimes b_{p}\right)=b_{1} \otimes \cdots \otimes b_{r-1} \otimes a b_{r} \otimes b_{r+1} \otimes \cdots \otimes b_{p}
$$

for all $b_{1}, \ldots, b_{p} \in A$.

Definition. Let $\operatorname{Der}^{p} A$ denote the vector space of $p$-linear maps $D$ : $A \times \cdots \times A \rightarrow \bigotimes^{p} A$ with the property that

$$
\begin{aligned}
D\left(a_{1}, \ldots, a_{r-1}, b c, a_{r+1}, \ldots\right. & \left.a_{p}\right) \\
= & Z_{b}^{r}\left(D\left(a_{1}, \ldots, a_{r-1}, c, a_{r+1}, \ldots, a_{p}\right)\right) \\
& +Z_{c}^{r}\left(D\left(a_{1}, \ldots, a_{r-1}, b, a_{r+1}, \ldots, a_{p}\right)\right)
\end{aligned}
$$

for every $r \in\{1, \ldots, p\}$ and all $a_{1}, \ldots, a_{r-1}, a_{r+1}, \ldots, a_{p}, b, c \in A$.

Note that $\operatorname{Der}^{0} A=\mathbb{R}$ and $\operatorname{Der}^{1} A=\operatorname{Der} A$.

Consider the tensor product $\bigotimes^{p}$ Der $A$ of the vector spaces Der $A$. We have the linear map $I^{p}: \bigotimes^{p} \operatorname{Der} A \rightarrow \operatorname{Der}^{p} A$ such that

$$
I^{p}\left(D_{1} \otimes \cdots \otimes D_{p}\right)\left(a_{1}, \ldots, a_{p}\right)=D_{1}\left(a_{1}\right) \otimes \cdots \otimes D_{p}\left(a_{p}\right)
$$

for all $D_{1}, \ldots, D_{p} \in \operatorname{Der} A$ and $a_{1}, \ldots, a_{p} \in A$.

Lemma. $I^{p}: \bigotimes^{p} \operatorname{Der} A \rightarrow \operatorname{Der}^{p} A$ is an isomorphism of vector spaces.

Proof. The proof is by induction on $p$. For $p=0$ and $p=1$ there is nothing to prove. Suppose $p \geq 2$ and the assertion of the Lemma is true for $p-1$. Let $D \in \operatorname{Der}^{p} A$.

Fix a basis $\varepsilon_{1}, \ldots, \varepsilon_{a}$ of the vector space $A$. For any $a_{1}, \ldots, a_{p-1}, b \in A$ there are unique $E_{b}^{i}\left(a_{1}, \ldots, a_{p-1}\right) \in \bigotimes^{p-1} A$ indexed by $i \in\{1, \ldots, a\}$ such that

$$
D\left(a_{1}, \ldots, a_{p-1}, b\right)=\sum_{i=1}^{a} E_{b}^{i}\left(a_{1}, \ldots, a_{p-1}\right) \otimes \varepsilon_{i} .
$$

From the uniqueness of $E_{b}^{i}\left(a_{1}, \ldots, a_{p-1}\right)$ we easily deduce that $E_{b}^{i} \in \operatorname{Der}^{p-1} A$ for every $i \in\{1, \ldots, a\}$ and every $b \in A$.

Fix a basis $\partial_{1}, \ldots, \partial_{d}$ of the vector space Der $A$. By assumption, $I^{p-1}$ is an isomorphism, and so $I^{p-1}\left(\partial_{l_{1}} \otimes \cdots \otimes \partial_{l_{p-1}}\right)$ for $l_{1}, \ldots, l_{p-1} \in\{1, \ldots, d\}$ form a basis of the vector space $\operatorname{Der}^{p-1} A$. Therefore for every $i \in\{1, \ldots, a\}$ and every $b \in A$ there are unique $F^{l_{1} \ldots l_{p-1} i}(b) \in \mathbb{R}$ indexed by $l_{1}, \ldots, l_{p-1} \in$ $\{1, \ldots, d\}$ such that

$$
E_{b}^{i}=\sum_{l_{1}=1}^{d} \ldots \sum_{l_{p-1}=1}^{d} F^{l_{1} \ldots l_{p-1} i}(b) I^{p-1}\left(\partial_{l_{1}} \otimes \cdots \otimes \partial_{l_{p-1}}\right),
$$

which is equivalent to

$$
E_{b}^{i}\left(a_{1}, \ldots, a_{p-1}\right)=\sum_{l_{1}=1}^{d} \ldots \sum_{l_{p-1}=1}^{d} F^{l_{1} \ldots l_{p-1} i}(b) \partial_{l_{1}}\left(a_{1}\right) \otimes \cdots \otimes \partial_{l_{p-1}}\left(a_{p-1}\right)
$$

for all $a_{1}, \ldots, a_{p-1} \in A$. 
Taking, for every $b \in A$,

$$
G^{l_{1} \ldots l_{p-1}}(b)=\sum_{i=1}^{a} F^{l_{1} \ldots l_{p-1} i}(b) \varepsilon_{i}
$$

we get a family of elements of $A$ indexed by $l_{1}, \ldots, l_{p-1} \in\{1, \ldots, d\}$ such that

$$
D\left(a_{1}, \ldots, a_{p-1}, b\right)=\sum_{l_{1}=1}^{d} \ldots \sum_{l_{p-1}=1}^{d} \partial_{l_{1}}\left(a_{1}\right) \otimes \cdots \otimes \partial_{l_{p-1}}\left(a_{p-1}\right) \otimes G^{l_{1} \ldots l_{p-1}}(b)
$$

for all $a_{1}, \ldots, a_{p-1} \in A$. Moreover, $G^{l_{1}, \ldots, l_{p-1}}(b)$ in this formula are uniquely determined, because of the uniqueness of $E_{b}^{i}\left(a_{1}, \ldots, a_{p-1}\right)$ and $F^{l_{1} \ldots l_{p-1}}(b)$. From the uniqueness of $G^{l_{1} \ldots l_{p-1}}(b)$ we easily deduce that $G^{l_{1} \ldots l_{p-1}} \in \operatorname{Der} A$ for all $l_{1}, \ldots, l_{p-1} \in\{1, \ldots, d\}$.

For any $l_{1}, \ldots, l_{p-1} \in\{1, \ldots, d\}$ there are unique $H^{l_{1} \ldots l_{p-1} m} \in \mathbb{R}$ indexed by $m \in\{1, \ldots, d\}$ such that

$$
G^{l_{1} \ldots l_{p-1}}=\sum_{m=1}^{d} H^{l_{1} \ldots l_{p-1} m} \partial_{m}
$$

Hence

$$
D\left(a_{1}, \ldots, a_{p}\right)=\sum_{l_{1}=1}^{d} \ldots \sum_{l_{p}=1}^{d} H^{l_{1} \ldots l_{p}} \partial_{l_{1}}\left(a_{1}\right) \otimes \cdots \otimes \partial_{l_{p}}\left(a_{p}\right)
$$

for all $a_{1}, \ldots, a_{p} \in A$, which is equivalent to

$$
D=\sum_{l_{1}=1}^{d} \ldots \sum_{l_{p}=1}^{d} H^{l_{1} \ldots l_{p}} I^{p}\left(\partial_{l_{1}} \otimes \cdots \otimes \partial_{l_{p}}\right)
$$

Moreover, $H^{l_{1} \ldots l_{p}}$ in these formulas are uniquely determined, because of the uniqueness of $G^{l_{1} \ldots l_{p-1}}(b)$ and $H^{l_{1} \ldots l_{p-1} m}$ in the previous formulas. This means that $I^{p}\left(\partial_{l_{1}} \otimes \cdots \otimes \partial_{l_{p}}\right)$ for $l_{1}, \ldots, l_{p} \in\{1, \ldots, d\}$ form a basis of $\operatorname{Der}^{p} A$, which implies that $I^{p}$ is an isomorphism. This completes the proof.

Let $e_{1}, \ldots, e_{n}$ denote the standard basis of $\mathbb{R}^{n}$. We will identify $A^{n}$ with $A \otimes \mathbb{R}^{n}$, and consequently $\bigotimes^{p} A^{n}$ with $\bigotimes^{p} A \otimes \bigotimes^{p} \mathbb{R}^{n}$. Hence (2) may be written as

$$
\widetilde{D}_{\mathbb{R}^{n}}(X)=\sum_{s=1}^{n} D\left(X^{s}\right) \otimes e_{s}
$$

for every $D \in \operatorname{Der} A$ and every $X \in A^{n}$.

We can now formulate our main result.

THEOREM. If $n \geq p$, then there is a one-to-one correspondence between the canonical tensor fields of type $(p, 0)$ on $T^{A}$ and the elements of $\operatorname{Der}^{p} A$. 
Namely, every $D \in \operatorname{Der}^{p} A$ induces a unique canonical tensor field $\widetilde{D}$ of type $(p, 0)$ on $T^{A}$ such that

$$
\widetilde{D}_{\mathbb{R}^{n}}(X)=\sum_{s_{1}=1}^{n} \ldots \sum_{s_{p}=1}^{n} D\left(X^{s_{1}}, \ldots, X^{s_{p}}\right) \otimes e_{s_{1}} \otimes \cdots \otimes e_{s_{p}}
$$

for every $X \in A^{n}$, and conversely, for every canonical tensor field $V$ of type $(p, 0)$ on $T^{A}$ there is a unique $D \in \operatorname{Der}^{p} A$ such that $V=\widetilde{D}$.

Proof. Let $D \in \operatorname{Der}^{p} A$. Our first task is to construct $\widetilde{D}$. Since we can use any chart on an $n$-dimensional manifold as $f$ in (1), we see that if $V, W$ are two canonical tensor fields of type $(p, 0)$ on $T^{A}$ such that $V_{\mathbb{R}^{n}}=W_{\mathbb{R}^{n}}$, then $V=W$. Hence (5) guarantees the uniqueness of $\widetilde{D}$. Let $\partial_{1}, \ldots, \partial_{d}$ be a basis of the vector space $\operatorname{Der} A$. By the lemma, there are $H^{l_{1} \ldots l_{p}} \in \mathbb{R}$ for $l_{1}, \ldots, l_{p} \in\{1, \ldots, d\}$ such that $(4)$ holds. Put

$$
\widetilde{D}_{M}=\sum_{l_{1}=1}^{d} \ldots \sum_{l_{p}=1}^{d} H^{l_{1} \ldots l_{p}} \widetilde{\partial_{l_{1} M}} \otimes \cdots \otimes \widetilde{\partial_{l_{p}}}
$$

for every $n$-dimensional manifold $M$. It is evident that this $\widetilde{D}$ is a canonical tensor field of type $(p, 0)$ on $T^{A}$. From (4) we conclude that it satisfies (5), and the construction of $\widetilde{D}$ is complete.

We now turn to the second part of the theorem. Fix a canonical tensor field $V$ of type $(p, 0)$ on $T^{A}$.

Since for every $X \in A^{n}$ we have $V_{\mathbb{R}^{n}}(X) \in \bigotimes^{p} A \otimes \bigotimes^{p} \mathbb{R}^{n}$, there are unique smooth $B^{s_{1} \ldots s_{p}}: A^{n} \rightarrow \bigotimes^{p} A$ for $s_{1}, \ldots, s_{p} \in\{1, \ldots, n\}$ such that

$$
V_{\mathbb{R}^{n}}(X)=\sum_{s_{1}=1}^{n} \ldots \sum_{s_{p}=1}^{n} B^{s_{1} \ldots s_{p}}(X) \otimes e_{s_{1}} \otimes \cdots \otimes e_{s_{p}}
$$

for every $X \in A^{n}$. Hence for every open subset $U$ of $\mathbb{R}^{n}$ and every embedding $f: U \rightarrow \mathbb{R}^{n}$ we can write (1) in the form

$$
\begin{aligned}
& \sum_{t_{1}=1}^{n} \cdots \sum_{t_{p}=1}^{n}\left(Z_{T^{A} \frac{\partial f^{s} 1}{\partial x^{t_{1}}}(X)}^{1} \circ \cdots \circ Z_{T^{A} \frac{\partial f^{s} p}{\partial x^{t} p}(X)}^{p}\right)\left(B^{t_{1} \ldots t_{p}}(X)\right) \\
&=B^{s_{1} \cdots s_{p}}\left(T^{A} f(X)\right)
\end{aligned}
$$

for all $s_{1}, \ldots, s_{p} \in\{1, \ldots, n\}$ and every $X \in T^{A} U$.

From (7) with

$$
f: \mathbb{R}^{n} \ni x \mapsto\left(\lambda^{1} x^{1}, \ldots, \lambda^{n} x^{n}\right) \in \mathbb{R}^{n},
$$

where $\lambda^{1}, \ldots, \lambda^{n} \in \mathbb{R} \backslash\{0\}$, we have

$$
\lambda^{s_{1}} \ldots \lambda^{s_{p}} B^{s_{1} \ldots s_{p}}(X)=B^{s_{1} \ldots s_{p}}\left(\lambda^{1} X^{1}, \ldots, \lambda^{n} X^{n}\right)
$$


for all $s_{1}, \ldots, s_{p} \in\{1, \ldots, n\}$ and every $X \in A^{n}$. By continuity, the same is true for all $\lambda^{1}, \ldots, \lambda^{n} \in \mathbb{R}$. The homogeneous function theorem (see [2]) now shows that for any $s_{1}, \ldots, s_{p} \in\{1, \ldots, n\}$ there is a $p$-linear $C^{s_{1} \ldots s_{p}}$ : $A \times \cdots \times A \rightarrow \bigotimes^{p} A$ such that

$$
B^{s_{1} \ldots s_{p}}(X)=C^{s_{1} \ldots s_{p}}\left(X^{s_{1}}, \ldots, X^{s_{p}}\right)
$$

for every $X \in A^{n}$.

From now on we use the assumption $n \geq p$. Put

$$
D=C^{1 \ldots p} .
$$

Let $r \in\{1, \ldots, p\}$. From (7) with $s_{1}=1, \ldots, s_{p}=p, U=\left\{x \in \mathbb{R}^{n}: x^{r}>0\right\}$ and

$$
f: U \ni x \mapsto\left(x^{1}, \ldots, x^{r-1},\left(x^{r}\right)^{2}, x^{r+1}, \ldots, x^{n}\right) \in \mathbb{R}^{n}
$$

we have

$$
2 Z_{X^{r}}^{r}\left(B^{1 \ldots p}(X)\right)=B^{1 \ldots p}\left(X^{1}, \ldots, X^{r-1},\left(X^{r}\right)^{2}, X^{r+1}, \ldots, X^{n}\right)
$$

for every $X \in T^{A} U$. This may be written as

$$
2 Z_{X_{r}}^{r}\left(D\left(X^{1}, \ldots, X^{p}\right)\right)=D\left(X^{1}, \ldots, X^{r-1},\left(X^{r}\right)^{2}, X^{r+1}, \ldots, X^{p}\right) .
$$

In the same manner, with $U$ replaced by $\left\{x \in \mathbb{R}^{n}: x^{r}<0\right\}$, we can see that (8) also holds for $X \in T^{A}\left\{x \in \mathbb{R}^{n}: x^{r}<0\right\}$, and so, by continuity, for every $X \in A^{n}$. Now the polarization of (8) with respect to $X^{r}$ shows that $D$ satisfies (3), and consequently $D \in \operatorname{Der}^{p} A$.

Our next goal is to show that

$$
C^{u_{1} \ldots u_{p}}\left(X^{u_{1}}, \ldots, X^{u_{p}}\right)=D\left(X^{u_{1}}, \ldots, X^{u_{p}}\right)
$$

for all $u_{1}, \ldots, u_{p} \in\{1, \ldots, n\}$ and every $X \in A^{n}$. We will identify any sequence $u_{1}, \ldots, u_{p} \in\{1, \ldots, n\}$ with $u:\{1, \ldots, p\} \rightarrow\{1, \ldots, n\}$ given by $u(1)=u_{1}, \ldots, u(p)=u_{p}$ and denote by $N(u)$ the number of elements of the set $u(\{1, \ldots, p\})$ for every such function $u$. The proof of (9) is by induction on $N(u)$. Fix $v:\{1, \ldots, p\} \rightarrow\{1, \ldots, n\}$ and suppose (9) holds whenever $N(u) \in\{N(v)+1, \ldots, p\}$. Choose a subset $R$ of $\{1, \ldots, p\}$ such that for each $r \in v(\{1, \ldots, p\})$ the set $v^{-1}(\{r\}) \cap R$ has one element. There is a bijective $w:\{1, \ldots, n\} \rightarrow\{1, \ldots, n\}$ such that $w|R=v| R$. Put

$$
S_{r}= \begin{cases}\left\{w_{r}\right\} & \text { if } r \in R \cup\{p+1, \ldots, n\}, \\ \left\{v_{r}, w_{r}\right\} & \text { if } r \in\{1, \ldots, p\} \backslash R .\end{cases}
$$

From (7) with $s_{1}=1, \ldots, s_{p}=p$ and

$$
f: \mathbb{R}^{n} \ni x \mapsto\left(\sum_{u_{1} \in S_{1}} x^{u_{1}}, \ldots, \sum_{u_{n} \in S_{n}} x^{u_{n}}\right) \in \mathbb{R}^{n}
$$

we have

$$
\sum_{u_{1} \in S_{1}} \ldots \sum_{u_{p} \in S_{p}} B^{u_{1} \ldots u_{p}}(X)=B^{1 \ldots p}\left(\sum_{u_{1} \in S_{1}} X^{u_{1}}, \ldots, \sum_{u_{n} \in S_{n}} X^{u_{n}}\right)
$$


for every $X \in A^{n}$. This may be written as

$$
\begin{aligned}
\sum_{u_{1} \in S_{1}} \ldots \sum_{u_{p} \in S_{p}} C^{u_{1} \ldots u_{p}}\left(X^{u_{1}}, \ldots, X^{u_{p}}\right) & \\
& =\sum_{u_{1} \in S_{1}} \ldots \sum_{u_{p} \in S_{p}} D\left(X^{u_{1}}, \ldots, X^{u_{p}}\right) .
\end{aligned}
$$

But if $u_{1} \in S_{1}, \ldots, u_{p} \in S_{p}$ are such that there is $r \in\{1, \ldots, p\} \backslash R$ with the property that $u_{r}=w_{r}$, then $C^{u_{1} \ldots u_{p}}\left(X^{u_{1}}, \ldots, X^{u_{p}}\right)=D\left(X^{u_{1}}, \ldots, X^{u_{p}}\right)$, on account of our assumption, because $N(u) \in\{N(v)+1, \ldots, p\}$ in this case. Subtracting all terms with such indices $u_{1}, \ldots, u_{p}$ from each side of (10) gives $C^{v_{1} \ldots v_{p}}\left(X^{v_{1}}, \ldots, X^{v_{p}}\right)=D\left(X^{v_{1}}, \ldots, X^{v_{p}}\right)$, which is due to the fact that $w|R=v| R$. This completes the proof of (9).

Applying (9) we can rewrite (6) as

$$
V_{\mathbb{R}^{n}}(X)=\sum_{s_{1}=1}^{n} \ldots \sum_{s_{p}=1}^{n} D\left(X^{s_{1}}, \ldots, X^{s_{p}}\right) \otimes e_{s_{1}} \otimes \cdots \otimes e_{s_{p}}
$$

for every $X \in A^{n}$. Hence $V_{\mathbb{R}^{n}}=\widetilde{D}_{\mathbb{R}^{n}}$, which yields $V=\widetilde{D}$ on account of the above remark. The uniqueness of $D$ is obvious. This completes the proof.

Combining the lemma with the theorem we obtain the following corollary.

Corollary. If $n \geq p$, then every canonical tensor field of type $(p, 0)$ on $T^{A}$ is a sum of tensor products of canonical vector fields on $T^{A}$. More precisely, if $\partial_{1}, \ldots, \partial_{d}$ is a basis of the vector space Der $A$, then for every canonical tensor field $V$ of type $(p, 0)$ on $T^{A}$ there are unique $H^{l_{1} \ldots l_{p}} \in \mathbb{R}$ for $l_{1}, \ldots, l_{p} \in\{1, \ldots, d\}$ such that

$$
V_{M}=\sum_{l_{1}=1}^{d} \cdots \sum_{l_{p}=1}^{d} H^{l_{1} \ldots l_{p}} \widetilde{\partial_{l_{1}}} \otimes \cdots \otimes \widetilde{\partial_{l_{p}}}
$$

for every $n$-dimensional manifold $M$.

The remainder of the paper is devoted to the following example.

ExAmple. Consider the Weil algebra $\mathbb{D}_{k}^{r}$ of $r$-jets at 0 of smooth functions $\mathbb{R}^{k} \rightarrow \mathbb{R}$, where $r$ and $k$ are non-negative integers.

We will denote by $x^{i}$ for $i \in\{1, \ldots, k\}$ the $r$-jet at 0 of the $i$ th projection $\mathbb{R}^{k} \rightarrow \mathbb{R}$ and write $x^{\alpha}=\left(x^{1}\right)^{\alpha^{1}} \cdots\left(x^{k}\right)^{\alpha^{k}}$ and $|\alpha|=\alpha^{1}+\cdots+\alpha^{k}$ for $\alpha \in \mathbb{N}^{k}$, where $\mathbb{N}$ stands for the set of non-negative integers. In addition, let $e_{1}, \ldots, e_{k}$ denote the standard basis of the module $\mathbb{Z}^{k}$.

Let $D \in \operatorname{Der} \mathbb{D}_{k}^{r}$. For every $\alpha \in \mathbb{N}^{k}$,

$$
D\left(x^{\alpha}\right)=\sum_{i \in\left\{l \in\{1, \ldots, k\}: \alpha^{l}>0\right\}} \alpha^{i} x^{\alpha-e_{i}} D\left(x^{i}\right),
$$


as is easy to check by induction on $|\alpha|$. Of course, for every $i \in\{1, \ldots, k\}$ there are unique $K_{\alpha}^{i} \in \mathbb{R}$ indexed by $\alpha \in \mathbb{N}^{k}$ such that $|\alpha| \leq r$, for which

$$
D\left(x^{i}\right)=\sum_{\alpha \in\left\{\beta \in \mathbb{N}^{k}:|\beta| \leq r\right\}} K_{\alpha}^{i} x^{\alpha} .
$$

Since $\left(x^{i}\right)^{r+1}=0$, from $(11)$ it follows that $0=(r+1)\left(x^{i}\right)^{r} D\left(x^{i}\right)$. Combining this with (12) yields $(r+1) K_{0}^{i}\left(x^{i}\right)^{r}=0$, and so $K_{0}^{i}=0$. Hence (12) can be rewritten as

$$
D\left(x^{i}\right)=\sum_{\alpha \in\left\{\beta \in \mathbb{N}^{k}: 1 \leq|\beta| \leq r\right\}} K_{\alpha}^{i} x^{\alpha} .
$$

Conversely, let $K_{\alpha}^{i} \in \mathbb{R}$ for $(i, \alpha) \in\{1, \ldots, k\} \times\left\{\beta \in \mathbb{N}^{k}: 1 \leq|\beta| \leq r\right\}$. We prove that there is a unique $D \in \operatorname{Der} \mathbb{D}_{k}^{r}$ such that (13) holds. Obviously, we define $D\left(x^{i}\right)$ for $i \in\{1, \ldots, k\}$ by (13), and next $D\left(x^{\alpha}\right)$ for $\alpha \in \mathbb{N}^{k}$ such that $|\alpha| \leq r$ by (11). Thus we have defined a linear $D: \mathbb{D}_{k}^{r} \rightarrow \mathbb{D}_{k}^{r}$, because the $x^{\alpha}$ for $\alpha \in \mathbb{N}^{k}$ such that $|\alpha| \leq r$ form a basis of the vector space $\mathbb{D}_{k}^{r}$. We only need to show that

$$
D\left(x^{\gamma} x^{\delta}\right)=x^{\gamma} D\left(x^{\delta}\right)+x^{\delta} D\left(x^{\gamma}\right)
$$

for all $\gamma, \delta \in \mathbb{N}^{k}$ such that $|\gamma| \leq r,|\delta| \leq r$. If $|\gamma+\delta| \leq r$, then $x^{\gamma} x^{\delta}=x^{\gamma+\delta}$ is an element of the basis in question, so we may use (11) three times to verify (14) in this case. Clearly, the left hand side of (14) vanishes whenever $|\gamma+\delta| \geq r+1$. If $|\gamma+\delta| \geq r+2$, then the right hand side vanishes on account of (11). If $|\gamma+\delta|=r+1$, then the right hand side also vanishes on account of (11) and the fact that the term $K_{0}^{i}$ is excluded from (13) for every $i \in\{1, \ldots, k\}$. This completes the proof.

Summing up, the map $J:$ Der $\mathbb{D}_{k}^{r} \rightarrow \mathbb{R}^{\{1, \ldots, k\} \times\left\{\beta \in \mathbb{N}^{k}: 1 \leq|\beta| \leq r\right\}}$ defined by the requirement that

$$
D\left(x^{i}\right)=\sum_{\alpha \in\left\{\beta \in \mathbb{N}^{k}: 1 \leq|\beta| \leq r\right\}} J(D)(i, \alpha) x^{\alpha}
$$

for all $D \in \operatorname{Der} \mathbb{D}_{k}^{r}$ and $i \in\{1, \ldots, k\}$ is an isomorphism of vector spaces. Counting elements of the set $\{1, \ldots, k\} \times\left\{\beta \in \mathbb{N}^{k}: 1 \leq|\beta| \leq r\right\}$ we obtain

$$
\operatorname{dim} \operatorname{Der} \mathbb{D}_{k}^{r}=k\left(\left(\begin{array}{c}
r+k \\
k
\end{array}\right)-1\right) .
$$

The above corollary now asserts that if $n \geq p$, then the dimension of the vector space of canonical tensor fields of type $(p, 0)$ on $T^{\mathbb{D}_{k}^{r}}$ equals

$$
\left(k\left(\left(\begin{array}{c}
r+k \\
k
\end{array}\right)-1\right)\right)^{p} .
$$

Of course, a canonical tensor field $V$ of type $(p, 0)$ on $T^{A}$ is called symmetric or skew-symmetric if the tensor field $V_{M}$ is symmetric or skew-symmetric 
for every $n$-dimensional manifold. Therefore if $n \geq p$, then the dimensions of the vector spaces of symmetric and skew-symmetric canonical tensor fields of type $(p, 0)$ on $T^{\mathbb{D}_{k}^{r}}$ equal

$$
\left(\begin{array}{c}
k\left(\left(\begin{array}{c}
r+k \\
k
\end{array}\right)-1\right)+p-1 \\
p
\end{array}\right) \text { and }\left(\begin{array}{c}
\left.k\left(\begin{array}{c}
r+k \\
k
\end{array}\right)-1\right) \\
p
\end{array}\right)
$$

respectively.

\section{References}

[1] I. Kolář, On the natural operators on vector fields, Ann. Global Anal. Geom. 6 (1988), $109-117$.

[2] I. Kolář, P. W. Michor and J. Slovák, Natural Operations in Differential Geometry, Springer, Berlin, 1993.

Institute of Mathematics

Jagiellonian University

Reymonta 4

30-059 Kraków, Poland

E-mail: debecki@im.uj.edu.pl 